\section{Conjugation of Cholic Acid in Human Liver Homogenates}

\author{
Urs Gloor and Per·Hugo Ekdahl
}

Department of Physiological Chemistry and Department of Surgery, University of Lund, Lund, Sweden

Cholic acid is the end-product of cholesterol metabolism, that is, no further oxidation of the steroid nucleus takes place. We therefore used it as a model substance for conjugation studies. In liver homogenates human bile acids are conjugated with taurine and glycine in different proportions ${ }^{1}$.

We have now developed this procedure further both as a macro and a micro method for homogenizing human liver biopsies. For macro experiments we used $1 \mathrm{ml} 20 \%$ homogenate in a medium similar to that described by Bucher ${ }^{2}$ containing $11 \mathrm{~g} \mathrm{KH} \mathrm{KHO}_{4}, 4 \mathrm{~g} \mathrm{KOH}$ (85\% pure), $1 \mathrm{~g} \mathrm{MgCl}, 6 \mathrm{H}_{2} \mathrm{O}$ and $3.7 \mathrm{~g}$ nicotinamide per liter. The $\mathrm{pH}$ was 7.4. A loose fitting pestle and a short homogenisation time are essential. We used a gap between tube and pestle of $1 \mathrm{~mm}$, a motor of 1500 RPM and 1 min homogenisation time. To $1 \mathrm{ml}$ medium we added $100 \mu \mathrm{g}(=40000$ counts per minute, end window tube) cholic acid-24. ${ }^{14} \mathrm{C}$ and $2 \mathrm{mg}$ adenosine triphosphate (crystalline, Pabst Laboratories, Milwaukee 5, Wisc.) and incubated in air for 2 hours at $37^{\circ}$ after which time we stopped the reaction by adding 5 volumes of ethanol and proceeded as described earlier ${ }^{3}$. The time between surgical operation and incubation of the homogenate should not exceed 2 hours and care has to be taken that no part of the liver piece becomes dry. For micro ex. periments, that is liver pieces obtained by punction through the abdominal wall, we used the same buffer solution and $15 \mu \mathrm{g}$ cholic acid $+200 \mu \mathrm{g}$ adenosine triphosphate in a total of $0.25 \mathrm{ml}$. The liver $(20-40 \mathrm{mg})$ was transferred directly from the biopsy needle into the homogenisation tube where all further operations were performed. We proceeded with methods similar to those described earlier ${ }^{3}$.

Some results are compiled in Table 1. Glyco+ tauro-conjugation $=100 \%$. The variations of the extent of conjugation $(1-50 \%)$ had no influence on the ratio between glycine and taurine coupling.

The patients in all three of the groups had different clinical test values and exact diagnosis may result in a further specification. Yet, the values for patients with cirrhosis are much lower than for the other groups. In this group

Table 1. Per cent glyco-cholic acid formed of total conjugated acids from $1 \mathrm{ml} 20 \%$ human liver homogenate with different patients.

Diagnos is

\begin{tabular}{ccc}
\hline $\begin{array}{c}\text { Liver cirrhosis } \\
\text { shunt } \\
\text { operations }\end{array}$ & $\begin{array}{c}\text { Gall-bladder } \\
\text { and bile } \\
\text { duct diseases }\end{array}$ & $\begin{array}{c}\text { Ulcus duodeni } \\
\text { Ulcus } \\
\text { ventriculi }\end{array}$ \\
\hline & & \\
10 & 43 & 57 \\
18 & 43 & 35 \\
25 & 40 & 54 \\
16 & 25 & 54 \\
17 & 47 & 56 \\
28 & 46 & 46 \\
& 39 & 36 \\
& 45 & 58 \\
& 48 & 57 \\
& 45 & 47 \\
\hline
\end{tabular}

a deficiency of glycine could be at least a partial explanation. In two experiments glycine and taurine (5 mole per mole acid) alone or together were added. In the case of single additions glyco- respectively tauro-conjugation was increased, whereas with both together the ratio between the conjugates did not differ from that without amino acids added.

In a fow cases we made macro and micro experiments from the same liver and found the results the same within limits of $\pm 10 \%, i$. $e$. the same deviation as among different macro experiments on the same liver.

1. Bergström, S. and Gloor, U. Acta Chem. Scand. 8 (1954) 1109.

2. Bucher, N. L. R. J. Am. Chem. Soc. 75 (1953) 498.

3. Bergström, S. and Gloor, U. Acta Chem. Scand. 8 (1954) 1373. 